UDC 514.74

\title{
INTERPOLATION BY RATIONAL SURFASES OF BEZIER AND NURBS-SURFASES
}

\author{
Badayev Yu.I., Doctor of Technical Sciences, \\ Lagodina L.P., Ph.D. \\ National Technical University of Ukraine «KPI name Igor Sikorsky» (Kyiv, \\ Ukraine)
}

Relevance. Rational Bezier surfases and NURBS-surfases are widely used in modeling curviliniar objects due to the great flexibility and efficiency of the method. Therefore, it is sense to develop an interpolation method by these surfases

Method. The work is devoted to the development of a new approach to interpolation surfases, represented by a set of discret points. The analytical description of the desired surfases is implemented a rational Bezier surfases and a NURBS-surfases.To solve this problem, two approaches are propozed. The first approach is that the weights of the control points are set in advance and then the coordinates of the points of the interpolating rational Bezier surfase as well as the NURBS-surfase are calculated. The second approach is that the coordinates of the control points are set in advance and then the weights of the control points of Bezier surfase as well as the NURBS-surfase are calculated. At the beginning of the process, are set only coordinates, but also parameters are set to a discret points, that is, each poins has the following definition: $T(x, y, z, u, v)$ in the three-dimentional space, where $u, v$ - parameters. To solve the interpolation problem, a system of linear equation is created in with each equation reflects the equality between the analytical formula for a surfase and a given point. Moreover, the number of interpolated points it must be number of control points. Thus, we have a system of $N$ linear equations, where $N$ is the number of control points.

Results. Two methods of interpolation of a points serials by rational Bezier surfases and NURBS-surfases. were developed.

Conclusions. The test cases carried out of using computer programs and calculated of results confirm the validiti of the proposed methods.

Keywords: interpolation, rational Bezier surfases, NURBS-surfases.

Formulation of the problem. To date, when constructing surfases in various automation systems, rational Bezier surfases and NURBS surfases are often used. It is a very flexible tool that allows you to create smooth splines of any order, shapes, and also easy to carry out local control over 
the surfase.

The surfase is represented in a parametric form and for controlling the shape of the surfase using control points and weight coefficients of nodes [1].

Rational nonuniform Bezier surfases refer to NURBS and are based on Bernshtein's basic functions. The practical application of NURBS surfases is very diverse, for example: they are often used in computer graphics to draw smooth surfases that accurately describe the shape of the three-dimensional objects depicted in drawings, for the task of the surfases of building the surfaces of rotation, as well as the simulation of the trajectories of motion on surfase and space in the course of time.The parametric representation of the surfases allows it to be used in multidimensional spaces. Interpolation of these surfases enables them to be used in modeling objects with complex geometric shapes.

Analysis of recent publications. In work [1] on page 135 offers an algorithm for interpolation with NURBS curves, which is based on the fact that control points in the second stage are projected onto a curve and these new points are taken as new control points. In practice, such an algorithm is difficult to implement and achieves precise results. In the works [2] and [3] it does not specify how to calculate the weight of control points, which prevents the construction of an algorithm for interpolation.

The purpose of the article. The purpose of the article is to develop real algorithms for interpolation with rational Bezier surfases and NURBS surfases for a given point series.

\section{Main part.}

The Rational surface of Besier is determined by a formula [1]:

$$
r=\frac{\sum_{i=0}^{n} \sum_{j=0}^{m} B_{i}^{n}(v) B_{j}^{m}(u) w_{i j} p_{i j}}{\sum_{i=0}^{n} \sum_{j=0}^{m} B_{i}^{n}(v) B_{j}^{m}(u) w_{i j}},
$$

where

$$
\begin{aligned}
& B_{i}^{n}(v)=\sum_{i=0}^{n} K_{i}^{n} v^{i}(1-v)^{(n-i)} \\
& B_{j}^{m}(\mathrm{u})=\sum_{j=0}^{m} K_{j}^{m} u^{j}(1-u)^{(\mathrm{m}-j)} \\
& K_{i}^{n}=\frac{n !}{i !(\mathrm{n}-\mathrm{i}) !} \text { - binomial coefficient; } \\
& p \text { - control points of surface; } \\
& w \text { - weight control points of surface. }
\end{aligned}
$$


The NURBS-surfase is determinated by the formula:

$$
r=\frac{\sum_{i=0}^{n} \sum_{j=0}^{m} N_{i k}^{n}(v) \mathrm{N}_{j p}^{m}(u) w_{i j} p_{i j}}{\sum_{i=0}^{n} \sum_{j=0}^{m} N_{i k}^{n}(v) \mathrm{N}_{j p}^{m}(u) w_{i j}},
$$

where $N_{i k}(u)$ normalized basic power function degrees $\mathrm{k}$.

Let given point series $\mathrm{R}_{l}, l=1,2,3, \ldots \mathrm{P}$. Acceptable for each point specific parameters $\mathrm{u}_{l}, \mathrm{v}_{l}$. We will have a point series $\mathrm{R}_{l}\left(\mathrm{x}_{l}, \mathrm{y}_{l}, \mathrm{z}_{l}, \mathrm{u}_{l}, \mathrm{v}_{l}\right)$. We will interpolate the given point series of the surfase (1). To do this we will create an equation system:

$$
\sum_{i=0}^{n} \sum_{j=0}^{m}\left[\frac{\sum_{i=0}^{n} \sum_{j=0}^{m} B_{i}^{n}(v) B_{j}^{m}(u) w_{i j} p_{i j}}{\sum_{i=0}^{n} \sum_{j=0}^{m} B_{i}^{n}(v) B_{j}^{m}(u) w_{i j}}-R_{l}\right]=0, i=0,1, \ldots n ; j=0,1, \ldots m .
$$

Or

$$
\begin{gathered}
\sum_{i=0}^{n} \sum_{j=0}^{m}\left[\sum_{i=0}^{n} \sum_{j=0}^{m} B_{i}^{n}(v) B_{j}^{m}(u) w_{i j} p_{i j}-\sum_{i=0}^{n} \sum_{j=0}^{m} B_{i}^{n}(v) B_{j}^{m}(u) w_{i j} R_{l}\right]=0, \\
i=0,1, \ldots n ; j=0,1, \ldots m .
\end{gathered}
$$

As you can see, the number of interpolated points should not exeed the number of control points of the surfase, i.e. $l=(n+1) x(m+1)$. Therefore, the same number of linear equations.

As an example, we take the rational Bezier surfase built by curves of the second degree:

$$
\begin{aligned}
& \mathrm{B}^{2}{ }_{0}=1.0, \\
& \mathrm{~B}^{2}{ }_{1}=2.0 \\
& \mathrm{~B}^{2}{ }_{2}=1.0 . \\
& \quad \sum_{i=0}^{n} \sum_{j=0}^{m} B_{i}^{n}(v) B_{j}^{m}(u) w_{i j}=w_{00}(1-v)^{2}\left[w_{00}(1-u)^{2}+2 w_{01}(1-u) u+w_{02} u^{2}\right] \\
& \quad+2 w_{10}(1-v) v\left[w_{10}(1-u)^{2}+2 w_{11}(1-u) u+w_{12} u^{2}\right] \\
& \quad+w_{20} v^{2}\left[w_{20}(1-u)^{2}+2 w_{21}(1-u) u+w_{22} u^{2}\right] .
\end{aligned}
$$

for

$$
\sum_{i=0}^{n} \sum_{j=0}^{m} B_{i}^{n}(v) B_{j}^{m}(u) w_{i j} p_{i j}
$$

will be the same as (4), but instead of $w_{i j}$ you need to set $\left(p_{i j} w_{i j}\right)$. 


\title{
Conclusions:
}

Two methods of interpolation of point row are worked out by the rational surface of Bezier and NURBS - surface.

Test examples by means of the computer program, that validify offer methods, are conducted.

\section{Referenses}

1. Голованов Н.Н. Геометрическое моделирование. М: Физматлит, 2002. 272c.

2. Юдин O.A. Интерполяция NURBS-кривыми в многомерном пространстве. Наукові ВНТУ, 2008. № 4. С.1-4.

3. Юдин О.А, Расширение интерполяции по Лагранжу с использованием кривых Безье: Нові технології, 2005. №3(9). C.117-120.

4. David F. Rodgers, Rae A. Earnshav(editor) «State of the Art in Computer Graphics»-Visualization and Modeling», 1991. P.225-269.

5. Кветний Р.Н. Методи комп'ютерних обчислень. Вінниця: ВДТУ, 2001. $148 \mathrm{c}$.

6. Гергель В.П. Теория и практика параллельных вычислений. М:Бином. Лаборатория знаний, 2007. 423c.

7. Бадаєв Ю.I., Блиндарук А.O. Керування кривиною NURBS-кривої 3-го порядку за допомогою ваги контрольних точок. Водний транспорт: зб. наукових праць Київської державної академії водного транспорту. 2014. №3(21). С.103-105.

8. Бадаєв Ю.І., Блиндарук А.О. Можливості локальної модифікації гладкої NURBS-кривої. Современные информационные $и$ электронные технологии: XV Международная научнопрактическая конференция: научн. труды. Одесса, 2014. Т.1. C. 26-27.

9. Бадаєв Ю.І., Блиндарук А.О. Комп'ютерна реалізація проектування криволінійних обводів методом NURBS-технологій вищих порядків. Сучасні проблеми моделювання. Технічні науки. Мелітополь, 2014. С.3-6.

\section{ІНТЕРПОЛЯЦІЯ РАЦІОНАЛЬНИМИ ПОВЕРХНЯМИ БЕЗЬС І NURBS-ПОВЕРХНЯМИ}

\author{
Бадаєв Ю.І. , Лагодіна Л.П.
}

Раціональні поверхні Безье $i$ NURBS-поверхні широко застосовуються в моделюванні криволінійних об'єктів завдяки великій гнучкості i працездатності метода. Тому актуальним є розробка 
методу інтерполячї цими поверхнями дискретного ряду точок в тривимірному просторі.

Робота присвячена розробиі нового підходу до інтерполяиії раціональною поверхнею Безьє, поданої множиною дискретних точок.

Аналітичний опис шуканої поверхні реалізується із застосуванням раціональної поверхні Безье $i$ NURBS-поверхні. Для розв'язання иієї задачі пропонується два підходи.

Перший підхід полягає в тому, щз заздалегідь задаються ваги контрольних точок $i$ далі розраховуються координати контрольних точок інтерполюючій рачіональній поверхні Безьє а також NURBSповерхні. Другий підхід полягає в тому, що заздалегідь задаються координати контрольних точок $i$ далі розраховуються ваги контрольних точок поверхні Безьє а також NURBS-поверхні.

На початку прочесу дискретному ряду точок задаються не тільки координати, але й також параметри, тобто кожна точка має наступне визначення: $T(x, y, z, u, v)$ в тривимірному просторi, де u,vпараметри. Для розв'язання задачі інтерполяиії створюється система лінійних рівнянь. в якому кожне рівняння відображає рівність між аналітичною формулою поверхні і заданою точкою.

При иъьому кількість інтерпольованих точок дорівнює кількості контрольних точок поверхні а також кількості лінійних рівнянь. Таким чином маємо систему із $N$ лінійних рівнянь, де $N$ - кількість інтерпольованих точок $i$ кількість контрольних точок поверхні. Невідомими є контрольні точки поверхні або в другому випадку ваги контрольних точок поверхні.

Розроблені два метода інтерполящіі точкового ряду раціональною поверхнею Безьє і NURBS-поверхнею.

Проведені тестові приклади за допомогою комп'ютерної програми, які підтверджують достовірність запропонованих мemodiв.

Ключові слова: інтерполячія, раціональні поверхні Безьє, NURBS- поверхні.

\title{
ИНТЕРПОЛЯЦИЯ РАЦИОНАЛЬНЫМИ ПОВЕРХНОСТЯМИ БЕЗЬЕ И NURBS-ПОВЕРХНОСТЯМИ.
}

\author{
Бадаев Ю.И., Лагодина Л.П.
}

Рачиональные поверхности Безье $u$ NURBS- поверхности широко применяются в моделировании криволинейных объектов благодаря большой гибкости и работоспособности метода. Поэтому 
актуальной является разработка метода интерполяции этими поверхностями дискретного ряда точек в трехмерном пространстве.

Работа посвящена разработке нового подхода к интерполячии рациональной поверхностью Безье, поданной множеством дискретных точек. Аналитическое описание искомой поверхности реализуется с применением рациональной поверхности Безье и NURBS- поверхности. Для решения этой задачи предлагается два nодхода.

Первый подход заключается в том, что заранее задаются веса контрольных точек $u$ дальше рассчитываются координаты контрольных точек интерполирующей рациональной поверхности Безье, а также NURBS- поверхности. Второй подход заключается в том, что загодя задаются координаты контрольных точек и дальше рассчитываются веса контрольных точек поверхности Безье, а также NURBS- поверхности.

В начале процесса дискретному ряду точек задаются не только координаты, но и также параметры, то есть каждая точка имеет следующее определение: $T(x, y, z, u, v)$ в трехмерном пространстве, где $u, v$ - параметры.

Для решения задачи интерполячии создается система линейных уравнений в котором каждое уравнение отображает равенство между аналитической формулой поверхности и заданной точкой.

При этом количество интерполируемых точек равняется количеству контрольных точек поверхности, а также количеству линейных уравнений. Таким образом имеем систему из $N$ линейных уравнений, где $N$ - количество интерполируемых точек и количество контрольных точек поверхности. Неизвестными являются контрольные точки поверхности или во втором случае веса контрольных точек поверхности.

Разработаны два метода интерполячии точечного ряда рациональной поверхностью Безье и NURBS- поверхностью.

Проведены тестовые примеры с помощью компьютерной программы, которые подтверждают достоверность предложенных методов.

Ключевые слова: интерполяция, рациональные поверхности Безье, NURBS - поверхности. 